\title{
Hybrid Energy Cell for Degradation of Methyl Orange by Self- Powered Electrocatalytic Oxidation
}

\author{
Ya Yang, ${ }^{\dagger, \perp}$ Hulin Zhang, ${ }^{\dagger, \perp}$ Sangmin Lee, ${ }^{\dagger}$ Dongseob Kim, ${ }^{\S}$ Woonbong Hwang, ${ }^{\S}$ \\ and Zhong Lin Wang ${ }^{\dagger,+, *}$ \\ ${ }^{\dagger}$ School of Materials Science and Engineering, Georgia Institute of Technology, Atlanta, Georgia 30332-0245, United States \\ ${ }^{\ddagger}$ Beijing Institute of Nanoenergy and Nanosystems, Chinese Academy of Sciences, Beijing, China \\ ${ }^{\S}$ Department of Mechanical Engineering, Pohang University of Science and Technology, San 31, Hyoja, Namgu, Pohang, Gyungbuk \\ 790-784, Korea
}

\section{Supporting Information}

ABSTRACT: In general, methyl orange (MO) can be degraded by an electrocatalytic oxidation process driven by a power source due to the generation of superoxidative hydroxyl radical on the anode. Here, we report a hybrid energy cell that is used for a self-powered electrocatalytic process for the degradation of MO without using an external power source. The hybrid energy cell can simultaneously or individually harvest mechanical and thermal energies. The mechanical energy was harvested by the triboelectric nanogenerator (TENG) fabricated at the top by using a flexible

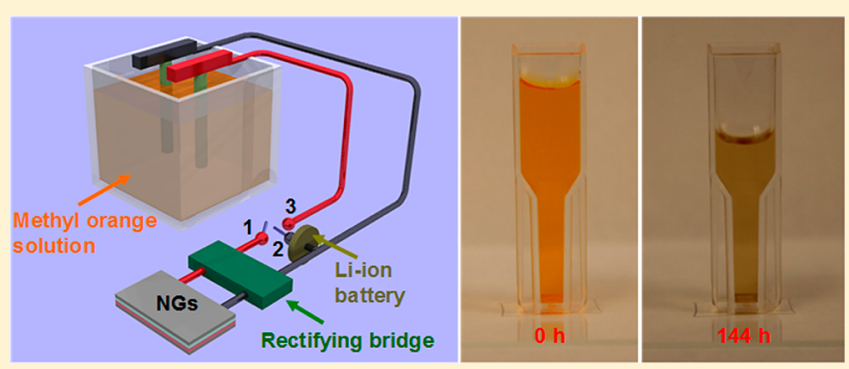
polydimethysiloxane (PDMS) nanowire array with diameters of about $200 \mathrm{~nm}$. A pyroelectric nanogenerator (PENG) was fabricated below the TENG to harvest thermal energy. The power output of the device can be directly used for electrodegradation of MO, demonstrating a self-powered electrocatalytic oxidation process.

KEYWORDS: Hybrid energy cell, triboelectric nanogenerator, pyroelectric nanogenerator, self-powered, methyl orange, electrodegradation

$\mathrm{W}$ ith the growing threat of pollution, global warming, and energy crises, the search for cost-effective, renewable and green energy sources to meet the global energy demands of the future is one of the most urgent challenges. ${ }^{1}$ Currently, there are three typical physics effects that can be used to fabricate nanogenerators (NGs) to harvest energy from the ambient environment. Both piezoelectric and triboelectric effect can be used to harvest the mechanical energy from irregular mechanical vibrations. ${ }^{2-5}$ The pyroelectric effect can be used to harvest the thermal energy from the time-dependent temperature fluctuations. $^{6-8}$ Since the mechanical and thermal energies are not always available at the same time in the environment, it is necessary to develop a hybrid energy cell technology to simultaneously/individually harvest both energies by using an integrated device. Although some attempts about the hybrid cells have been achieved, ${ }^{9-11}$ there has no report about a hybrid energy cell that consists of a triboelectric nanogenerator (TENG) and a pyroelectric nanogenerator (PENG) for harvesting mechanical and thermal energies.

The purpose of developing self-powered nanotechnology is to use NGs instead of batteries or other energy storage/supply systems to power small electronic devices (such as LCD, LED) or achieve some electrochemical applications (such as electrodeposition). ${ }^{12-14}$ Although some experiments about the selfpowered electrochemical applications have been demonstra- ted, ${ }^{14}$ there has no report about the self-powered degradation of dyeing wastewater by the electrocatalytic oxidation. Usually, wastewater from textile manufacturing contains dyes, which must be removed before it can be discharged. The purification of dyeing wastewater by electrochemical techniques has been proven to be a very effective method, where it has the simple equipment, easy operation, lower temperature requirements and no sludge formation. ${ }^{15-17}$ Although the feasibility of electrochemical degradation of dyes [such as methyl orange (MO), rhodamine $\mathrm{B}$ ] for wastewater treatment has been extensively reported, ${ }^{18-20}$ all these electrochemical investigations need external power sources, which largely limit the development of this technique. To solve this problem, the NGs may be used to achieve self-powered electrochemical degradation of dyeing wastewater by harvesting energy from environment. Usually, the mechanism of the electrodegradation of $\mathrm{MO}$ is based on an electrocatalytic oxidation by hydroxyl radical that is generated on the anode. ${ }^{21}$

Here, we demonstrated the first hybrid energy cell, which was used to simultaneously/individually harvest the mechanical and thermal energies for a self-powered electrodegradation of

Received: December 14, 2012

Revised: January 13, 2013 
MO. A TENG was fabricated at the top by using a flexible polydimethysiloxane (PDMS) nanowire array with the diameters of about $200 \mathrm{~nm}$ to harvest the mechanical energy, and a lead zirconate titanate (PZT) film-based pyroelectric nanogenerator (PENG) was fabricated at the bottom to harvest thermal energy. These energies produced by the NGs can be stored in a Li-ion battery or directly used for the degradation of MO by the self-powered electrocatalytic oxidation.

Figure 1a shows a schematic diagram of the fabricated hybrid energy cell. In this study, we used a homemade anodic

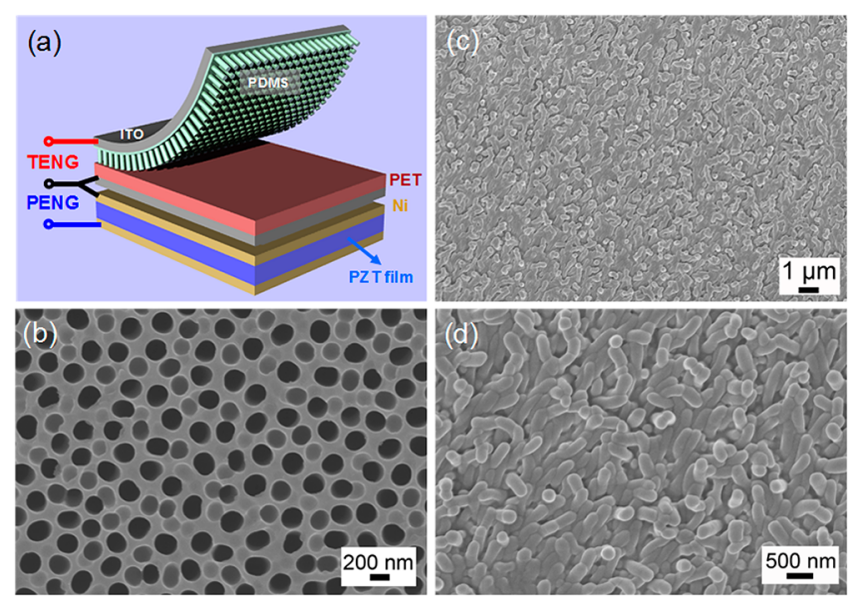

Figure 1. (a) Schematic diagram of the fabricated hybrid energy cell. (b) SEM image of the fabricated AAO template. (c) SEM image of the PDMS nanowire array. (d) Enlarged SEM image of the PDMS nanowire array.

aluminum oxide (AAO) template to fabricate the PDMS nanowire array. The top TENG consists of a transparent indium tin oxide (ITO) film electrode, the PDMS nanowire array layer of about $200 \mu \mathrm{m}$, and a polyester (PET) film of 127 $\mu \mathrm{m}$. The detailed fabrication methods of AAO template and PDMS nanowire array are given in Experimental Section. Figure $1 \mathrm{~b}$ shows a scanning electron microscopy (SEM) image of the fabricated AAO template, revealing that the diameters of the holes are about $200 \mathrm{~nm}$. The depth of the holes is about $500 \mathrm{~nm}$, as shown in Figure S1, Supporting Information. Figure 1c shows a SEM image of the obtained PDMS nanowire array, where the distribution of the nanowires is uniform. The enlarged SEM image indicates that the diameters of the PDMS nanowires are about $200 \mathrm{~nm}$, as shown in Figure 1d. Some nanowires are not vertical on the substrate, which is associated with the attraction forces among the PDMS nanowires when they were pulled out from the AAO template. The bottom PENG consists of two Ni electrodes and a PZT film with the thickness of about $1.5 \mathrm{~mm}$. The optical image of the fabricated hybrid energy cell was also shown in Figure S2, Supporting Information.

Parts $\mathrm{a}$ and $\mathrm{b}$ of Figure 2 show the output performance of the fabricated TENG under the forward and reversed connections, where the output voltage is up to $12 \mathrm{~V}$ and the output current is about $0.2 \mu \mathrm{A}$ (current density of $0.17 \mathrm{~mA} / \mathrm{m}^{2}$ ). The mechanism of the TENG is based on the electron flow as driven by the triboelectric effect induced electrostatic charges on the surfaces of the PDMS nanowire array and the PET film. ${ }^{5}$ In this study, when the PDMS is in contact with the PET film, the surface charges will be transferred from PET to PDMS due to the different triboelectric coefficients, resulting in the observed output signals. $^{22,23}$ The output voltage $V_{o c(T E N G)}$ of TENG can be given by

$$
V_{o c(T E N G)}=\frac{\sigma d}{\varepsilon_{0}}
$$

where $\sigma$ is the triboelectric charge density, $d$ is the interlayer distance, and $\varepsilon_{0}$ is the vacuum permittivity. ${ }^{24}$ According to eq 1 , the output voltage $V_{o c \text { (TENG) }}$ will increase with increasing the interlayer distance $d$.

The output performance of the PENG was measured by varying the temperature in the vicinity of the device from 295 to $309 \mathrm{~K}$, as shown in Figure 2c. The peak value of the temperature changing rate is about $0.8 \mathrm{~K} / \mathrm{s}$. The output current pulse of the PENG is about $0.3 \mu \mathrm{A}$ (current density of 0.33 $\mathrm{mA} / \mathrm{m}^{2}$ ) under the forward and reversed connections, as shown in Figure 2d. The corresponding output voltage pulse is about $16 \mathrm{~V}$, as shown in Figure S3, Supporting Information. The output voltage $V_{o c(P E N G)}$ of the PENG can be expressed as

$$
V_{o c(P E N G)}=\frac{p D \Delta T}{\left(\varepsilon_{r}-1\right) \varepsilon_{0}}
$$

where $p$ is pyroelectric coefficient, $D$ is the thickness of the device, $\Delta T$ is the change in temperature, and $\varepsilon_{\mathrm{r}}$ is the relative dielectric constant of the sample. ${ }^{25}$ According to eq 2, the output voltage of PENG will increase with increasing the change in temperature of the device.

Figure 3a shows the output current of the hybrid energy cell, where the two NGs were in parallel connection. It can be seen that the TENG and PENG can work simultaneously and individually to harvest mechanical and thermal energies, respectively. Since both the TENG and PENG have the alternating electric (ac) output signals, the total output current was sometimes weakened rather than enhanced (see the time from 400 to $550 \mathrm{~s}$ in Figure 3a). To solve this problem, these output signals need to be rectified by the full-wave bridge circuits, where the corresponding schematic diagram of the measurement circuit is shown in Figure S4, Supporting Information. Figure $3 \mathrm{~b}$ shows the output current of the hybrid energy cell after the output signals were rectified. It can be seen that all of the output current pulses are positive and the output current of the hybrid cell is always larger than that of the individual NGs.

To illustrate the potential applications of the hybrid energy cell, we demonstrated that the energies produced by the fabricated NGs can be used for the electrodegradation of MO. Figure $4 \mathrm{a}$ shows the schematic diagram of the self-powered degradation of $\mathrm{MO}$ by electrocatalytic oxidation. There are two methods to be used for the electrodegradation of MO. When the point " 1 " was connected to the point " 2 ", the energies can be stored in a Li-ion battery and then was used for the electrodegradation of MO. The second method is that the fabricated NGs were directly used for electrodegradation of MO when the point " 1 " was connected to the point " 3 ". In this study, the Pt electrode was used as the anode for electrocatalysis and $\mathrm{NaCl}$ as electrolyte was added into $\mathrm{MO}$ solution to improve the conductivity. The mechanism of the electrodegradation of $\mathrm{MO}$ is proposed as follows:

$$
\begin{aligned}
& 2 \mathrm{H}_{2} \mathrm{O} \rightarrow 2 \mathrm{OH}^{*}+2 \mathrm{H}^{+}+2 \mathrm{e}^{-} \\
& \mathrm{Cl}^{-}+\mathrm{H}_{2} \mathrm{O} \rightarrow \mathrm{ClO}^{-}+2 \mathrm{H}^{+}+2 \mathrm{e}^{-}
\end{aligned}
$$


Triboelectric Nanogenerator

(a)
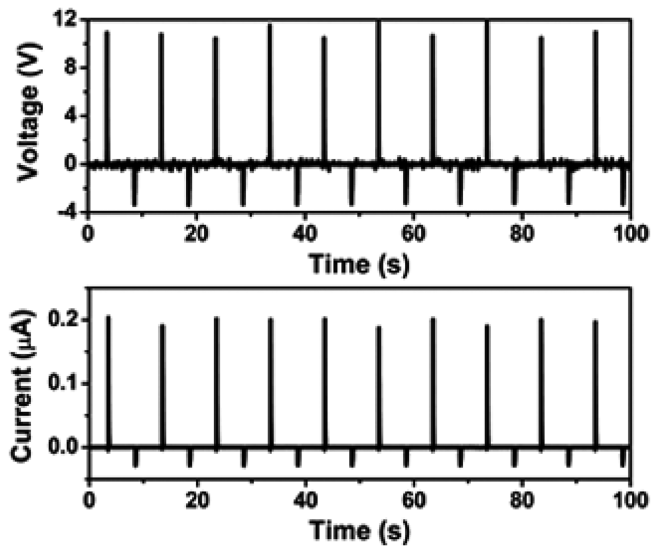

(b)
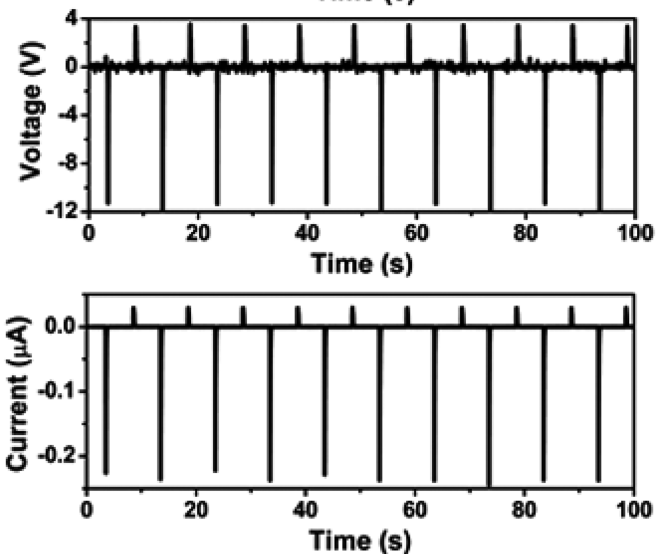

Pyroelectric Nanogenerator

(c)
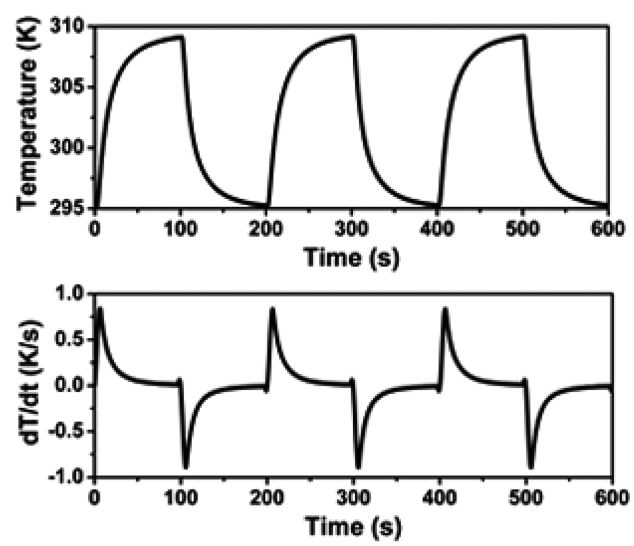

(d)
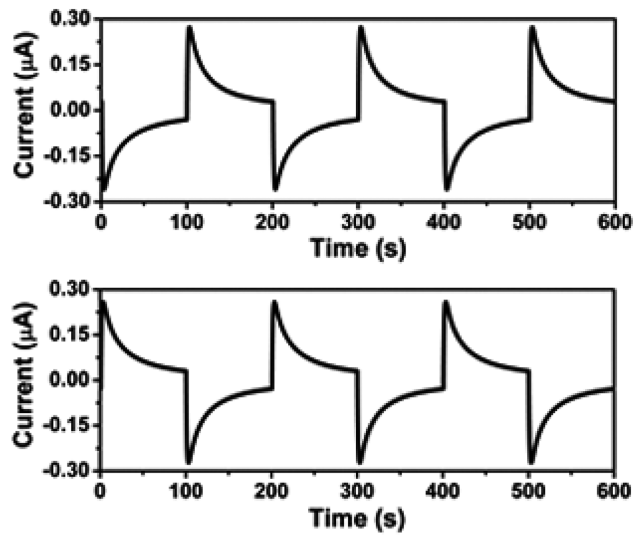

Figure 2. ( $a, b)$ The output voltage and current of the TENG under the forward connection (a) and the reversed connection (b) to the measurement system. (c) Cyclic change in temperature of the PENG and the corresponding differential curve. (d) Measured output current of the PENG under the forward and reversed connections.

According to eqs 3 and $4, \mathrm{H}_{2} \mathrm{O}$ and $\mathrm{Cl}^{-}$were adsorbed to $\mathrm{Pt}$ electrode (anode) by the discharge reaction of anode, resulting in the formation of superoxidative hydroxyl radical $\mathrm{OH}^{*}$ and $\mathrm{ClO}^{-}$due to the electrocatalysis of $\mathrm{Pt}^{26}$ Then the oxidative species can degrade $\mathrm{MO}$ into the small molecules like $\mathrm{CO}_{2}$, $\mathrm{H}_{2} \mathrm{O}$, etc. The electrodegradation of $\mathrm{MO}$ which proceeds via series of parallel and consecutive reactions is accomplished simultaneously by the redox processes. Also, a small amount of organic pollutants can be oxidized directly on the surface of $\mathrm{Pt}$ electrode.

Here, we chose the TENG as a model to demonstrate that the energy from NGs can be stored in a Li-ion battery and then was used for the electrodegradation of MO. According to eq 1 , Figure $4 \mathrm{~b}$ shows that the output voltage and current of the TENG were increased to $70 \mathrm{~V}$ and $1.8 \mu \mathrm{A}$ (current density of $0.28 \mathrm{~mA} / \mathrm{m}^{2}$ ), respectively. The structure of the TENG in Figure $4 \mathrm{~b}$ was the same as the one for Figure 2a, except the device size and the interlayer in eq 1 were increased, resulting in the increase of both the output voltage and current. A Li-ion battery can be charged by the TENG from about 0.18 to $1 \mathrm{~V}$, as shown in Figure 4c. Figure 4d shows that the UV-visible absorption spectra of the MO solution for electrodegradation by using a charged Li-ion battery of about $2 \mathrm{~V}$ at the same time intervals. The characteristic absorption peak of $\mathrm{MO}$, which is centered at $461 \mathrm{~nm}$, was selected for monitoring the electrodegradation process. It can be seen that the characteristic absorption peak intensity of MO decreases with increasing the electrodegradation time, indicating the decrease of the $\mathrm{MO}$ concentration.

In order to confirm that the decrease of MO absorption peak intensity is due to the electrodegradation further, a control experiment was finished, where no batteries or other power sources were used. Under this condition, there is no change in the absorption spectra of the MO solution at the same time intervals in Figure 4d, as shown in Figure 4e. The dynamic electrodegradation process of the MO solution was also shown in a movie file in the supporting file. It can be clearly seen that the electrodegradation process starts at the Pt anode electrode, where the color of the $\mathrm{MO}$ solution was changed from yellow to red and then to colorless. The change in color of the MO solution between red and yellow can be expressed as ${ }^{27}$

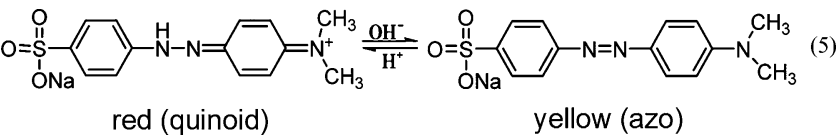

The existing form of $\mathrm{MO}$ is mainly $\mathrm{ArSO}_{3}{ }^{-}$anion, which has the color of yellow. According to eqs 3 and 4, there are many $\mathrm{H}^{+}$anions produced on the anode in the electrodegradation process, which can result in the change in color from yellow to red according to eq 5. The change in color of $\mathrm{MO}$ is consistent with the proposed mechanism of the electrodegradation of $\mathrm{MO}$ in the eqs 3-5. The optical images of the MO solution before and after degradation show an obvious difference (Figure S5, Supporting Information), indicating that the electrodegradation of $\mathrm{MO}$ is effective. 

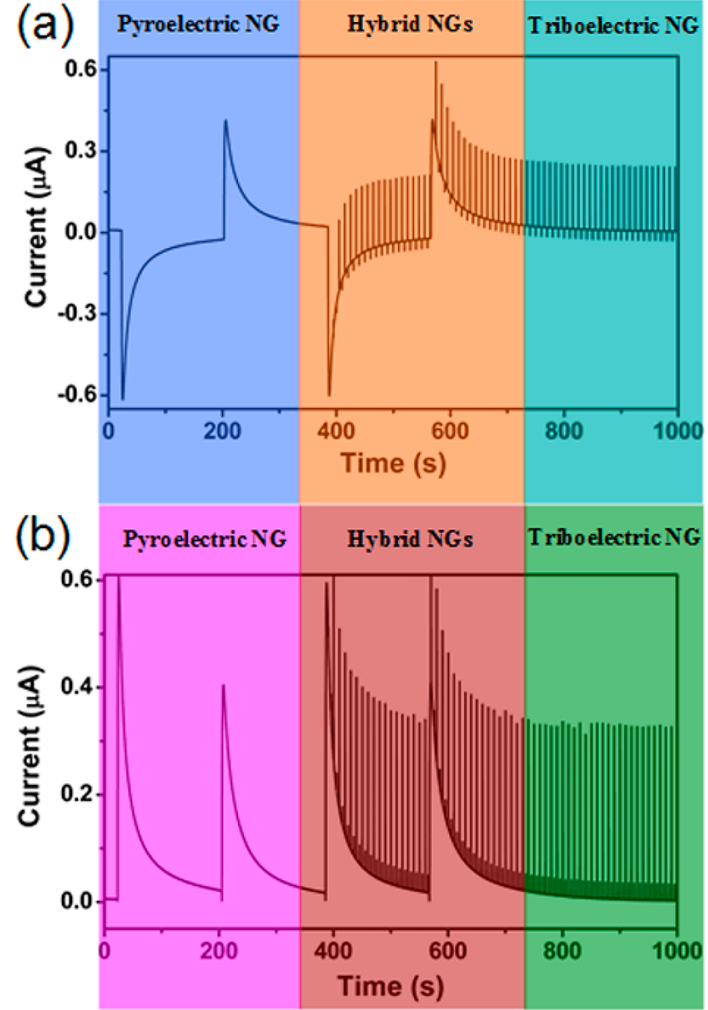

Figure 3. (a) Output current of the hybrid energy cell, where the TENG and PENG can work simultaneously and individually. (b) Output current of the hybrid energy cell after the output signals were rectified by a full-wave bridge circuit.
Without Li-ion batteries as the storage units, we also demonstrated the PENG as a model directly used for the electrodegradation of MO. Figure 5a shows the output performance of the PENG, where the output voltage and current are about $9 \mathrm{~V}$ and $0.4 \mu \mathrm{A}$, respectively. Figure $5 \mathrm{~b}$ shows that the UV-visible absorption spectra of the MO solution for electrodegradation by using PENG at the same time intervals. It can be seen that the characteristic absorption peak intensity of MO decreases with increasing the degradation time. The degradation percentage of $\mathrm{MO}$ is up to $80 \%$ after $144 \mathrm{~h}$, as shown in Figure 5c. The optical images of the MO solution before and after degradation are shown in the inset in Figure 5c. The color of MO solution at $144 \mathrm{~h}$ was obviously shallowed as compared with that before the degradation, indicating that the MO has been effectively degraded by using the PENG. Figure $5 \mathrm{~d}$ shows the change in maximum absorption peak of $\mathrm{MO}$ under the different degradation time, where the peak wavelength has been moved to the lower wavelength (a shift of about $80 \mathrm{~nm})$. It is associated with the higher voltage $(9 \mathrm{~V})$ produced by the PENG than that of the Li-ion battery $(2 \mathrm{~V})$. Usually, the certain characteristic peak corresponds to the certain chemical groups. Under the different voltages, the different intermediates can be induced in the electrocatalytic oxidation process, resulting in the shift of the absorption peak. $^{28}$

In summary, we have demonstrated a hybrid energy cell, which can simultaneously/individually harvest the mechanical and thermal energies for self-powered degradation of MO. By using the flexible PDMS nanowire array, the fabricated TENG at the top was used to harvest the mechanical energy, and a PZT film-based PENG at the bottom was used to harvest the
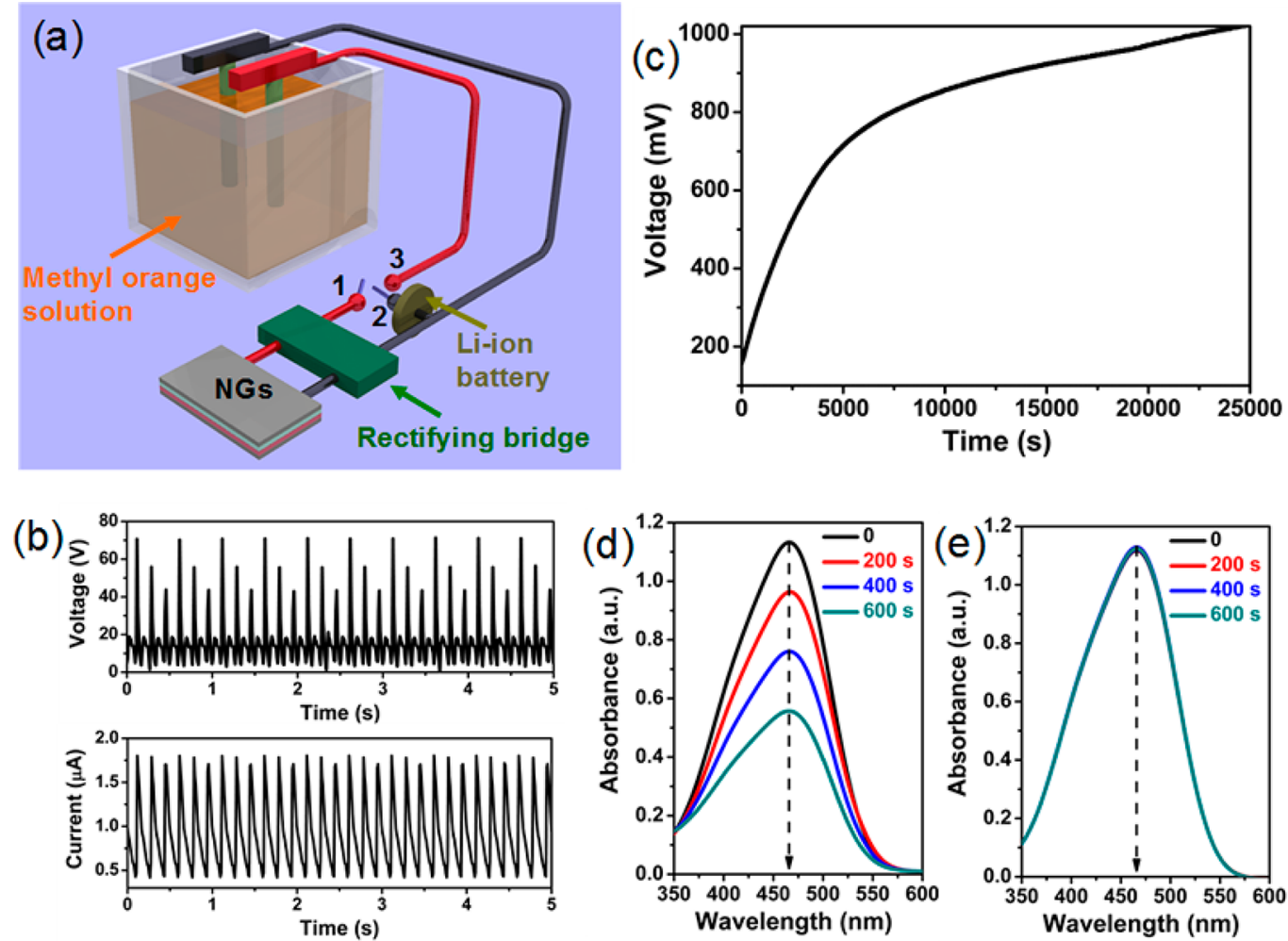

Figure 4. (a) Schematic diagram of the self-powered electrodegradation of MO. (b) Measured output voltage and current of the TENG. (c) Charging curve of a Li-ion battery by using the energy in part b. (d, e) Absorption spectra of the MO solution with (d) and without (e) a Li-ion battery of about $2 \mathrm{~V}$ under the same time intervals. 

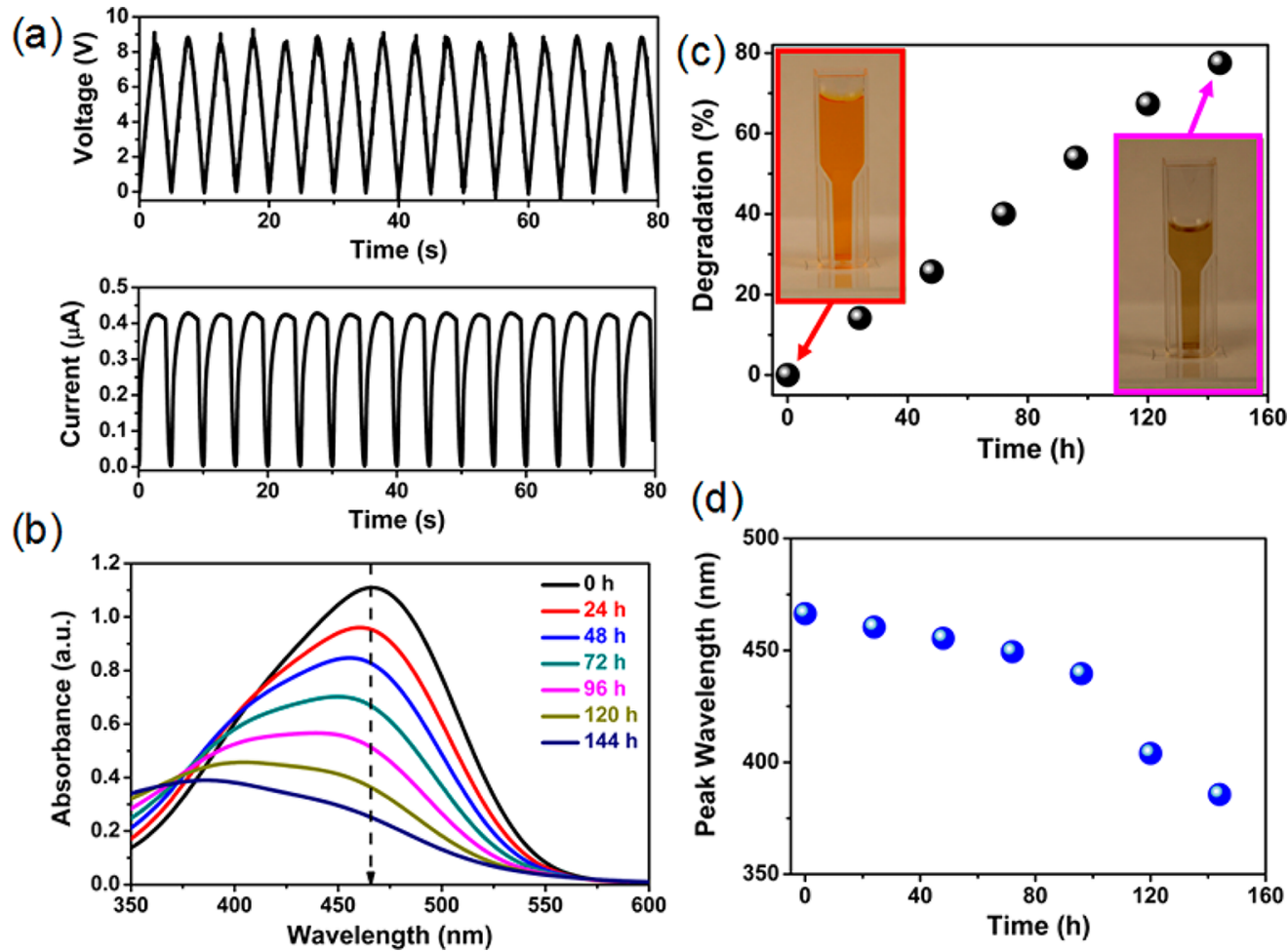

Figure 5. (a) Output voltage and current of the PENG. (b) Absorption spectra of the MO solution under the some time intervals. (c) Plot of degradation percentage versus the electrodegradation time. The inset shows the optical images of the MO solution before and after the electrodegradation. (d) Corresponding peak wavelength in $\mathrm{b}$ under the different electrodegradation time intervals.

thermal energy. The mechanical energy produced by the TENG was stored in a Li-ion battery, which can be used for degradation of $\mathrm{MO}$ by electrocatalytic oxidation. Moreover, the PENG was directly used for degradation of MO, where the degradation percentage is up to $80 \%$ after $144 \mathrm{~h}$. The fabricated hybrid energy cells have the potential electrochemical applications in the self-powered electrodeposition, pollutant degradation, corrosion protection, and water splitting.

\section{METHODS SUMMARY}

Fabrication of the AAO Template, the PDMS Nanowire Array, and the Hybrid Energy Cell. In order to fabricate nanoporous AAO substrates, industrial $\mathrm{Al}$ foils (99.5\%, $25 \mathrm{~mm}$ and $90 \mathrm{~mm}$ ) were prepared and anodized for $1 \mathrm{~h}$ in 0.1 $\mathrm{M}$ phosphoric acid at a constant voltage of $165 \mathrm{~V}$ using a direct current (dc) power supply (Digital electronics Co., DRP92001DUS). During the anodization, the solution was maintained at $275 \mathrm{~K}$ by a circulator (Lab. Companion, RW$0525 \mathrm{G})$. After the anodizing process, a widening process was performed in $0.1 \mathrm{M}$ phosphoric acid solution for $100 \mathrm{~min}$ at $303 \mathrm{~K}$ to enlarge the diameter of the nanoholes. The AAO substrates were washed by deionized water and dried at room temperature.

Then, in order to use the AAO substrate as a nanoimprinting template, the surface energy of the AAO substrate was lowered based on a self-assembled monolayer using a heptadecafluoro1,1,2,2-tetrahydrodecyl trichlorosilane (HDFS), which can reduce the surface adhesion between the AAO template and polymer materials. A mixture of $n$-hexane and HDFS was prepared in volumetric ratio of 1000:1. The AAO substrates were dipped in the solution for $5 \mathrm{~min}$ at room temperature and washed by $n$-hexane. PDMS was used as the nanoimprinted polymer and prepared by the gel-casting technique. The base monomer and curing agent (Sylgard 184, Dow corning) were mixed in mass ratio of 10:1. The mixed solution was evacuated for $30 \mathrm{~min}$ to remove the gas bubbles, and it was casted on to the surface of the HDFS-coated AAO substrate. The vacuum process was then used for $30 \mathrm{~min}$ to remove air remaining in the nanopores that formed in the AAO substrate. After that the substrate was kept in the oven at $358 \mathrm{~K}$ for $2 \mathrm{~h}$ to get cured, and the cured PDMS was peeled off from the AAO substrate.

The fabricated hybrid energy cell consists of a TENG and a PENG. A PET film with the ITO electrode was put above the PENG, and the PDMS nanowire array with the ITO electrode was put on the PET film. The TENG can work under the contact/separation between the PET film and the PDMS nanowire array. Then, a PZT film-based PENG was put on a thermoelectric-based heater, which was used to rapidly change the temperature of the device. A temperature sensor was used to record the temperature of PENG.

Measurement of the NGs and Electrodegradation of MO Solution. The output voltage of the NGs was measured by a low-noise voltage preamplifier (Keithley 6514 System Electrometer). The output current of the NGs was measured by a low-noise current preamplifier (Stanford Research SR560). The performance of the Li-ion battery was measured by a battery analyzer (MTI Corporation). The electrodegradation of MO was performed in a cuvette filled with $3 \mathrm{~mL}$ of $100 \mathrm{mg} / \mathrm{L}$ MO aqueous solution at ambient temperature. Prior to degradation, $0.1 \mathrm{~g}$ of $\mathrm{NaCl}$ as electrolyte was added into solution to improve the conductivity of the MO solution. Due to the good chemical stability and the high electrocatalytic activity, Pt electrode was used as anode, which was inserted into the MO solution. At each given time interval, the absorbance spectra of the MO solution was measured to determine the 
concentration change using an UV-visible spectrophotometer (JASCO V-630).

\section{ASSOCIATED CONTENT}

\section{S Supporting Information}

Additional figures showing the SEM image of the fabricated AAO template, the optical image of the fabricated device, the output voltage of the PENG under the forward and reversed connections, schematic diagram of the measurement circuit of the hybrid energy cell by using the full-wave bridge circuits, and the optical image of the MO solution before and after degradation, and a movie file showing the dynamic process of electrodegradation of MO by using a Li-ion battery, where the playback speed of the movie was increased by 10 times than the normal speed. This material is available free of charge via the Internet at http://pubs.acs.org.

\section{AUTHOR INFORMATION}

\section{Corresponding Author}

*E-mail: zlwang@gatech.edu.

\section{Author Contributions}

${ }^{\perp}$ These authors contributed equally to the work.

\section{Notes}

The authors declare no competing financial interest.

\section{ACKNOWLEDGMENTS}

This work was supported by Airforce, MURI, U.S. Department of Energy, Office of Basic Energy Sciences (DE-FG0207ER46394), NSF (CMMI 0403671), the Knowledge Innovation Program of the Chinese Academy of Sciences (KJCX2-YW-M13), and the National Research Foundation of Korea (NRF) grant funded by the Ministry of Education, Science, and Technology (MEST) (No. 2011-0018645).

\section{REFERENCES}

(1) Wang, Z. L.; Wu, W. Nanotechnology-Enabled Energy Harvesting for Self-Powered Micro-/Nanosystems. Angew. Chem. 2012, 51, 11700-11721.

(2) Chang, C.; Tran, V. H.; Wang, J.; Fuh, Y.-K.; Lin, L. Direct-Write Piezoelectric Polymeric Nanogenerator with High Energy Conversion Efficiency. Nano Lett. 2010, 10, 726-731.

(3) Kumar, B.; Lee, K. Y.; Park, H.-K.; Chae, S. J.; Lee, Y. H.; Kim, S.W. Controlled Growth of Semiconducting Nanowire, Nanowall, and Hybrid Nanostructures on Graphene for Piezoelectric Nanogenerators. ACS Nano 2011, 5, 4197-4204.

(4) Chen, X.; Xu, S.; Yao, N.; Shi, Y. 1.6 V Nanogenerator for Mechanical Energy Harvesting Using PZT Nanofibers. Nano Lett. 2010, 10, 2133-2137.

(5) Fan, F.-R.; Tian, Z.-Q.; Wang, Z. L. Flexible Triboelectric Generator. Nano Energy 2012, 1, 328-334.

(6) Lang, S. B. Pyroelectricity: From Ancient Curiosity to Modern Imaging Tool. Phys. Today 2005, 58, 31-36.

(7) Yang, Y.; Guo, W.; Pradel, K. C.; Zhu, G.; Zhou, Y.; Zhang, Y.; Hu, Y.; Lin, L.; Wang, Z. L. Pyroelectric Nanogenerators for Harvesting Thermoelectric Energy. Nano Lett. 2012, 12, 2833-2838.

(8) Yang, Y.; Jung, J. H.; Yun, B. K.; Zhang, F.; Pradel, K. C.; Guo, W.; Wang, Z. L. Flexible Pyroelectric Nanogenerators Using a Composite Structure of Lead-Free $\mathrm{KNbO}_{3}$ Nanowires. Adv. Mater. 2012, 24, 5357-5362.

(9) Xu, C.; Wang, X.; Wang, Z. L. Nanowire Structured Hybrid Cell for Concurrently Scavenging Solar and Mechanical Energies. J. Am. Chem. Soc. 2009, 131, 5866-5872.

(10) Hansen, B. J.; Liu, Y.; Yang, R.; Wang, Z. L. Hybrid Nanogenerator for Concurrently Harvesting Biomechanical and Biochemical Energy. ACS Nano 2010, 4, 3647-3652.
(11) Xu, C.; Wang, Z. L. Compacted Hybrid Cell Made by Nanowire Convoluted Structured for Harvesting Solar and Mechanical Energies. Adv. Mater. 2011, 23, 873-877.

(12) Xu, S.; Qin, Y.; Xu, C.; Wei, Y.; Yang, R.; Wang, Z. L. SelfPowered Nanowire Devices. Nat. Nanotechnol. 2010, 5, 366-373.

(13) Wang, Z. L. Toward Self-Powered Sensor Networks. Nano Today 2010, 5, 512-514.

(14) Guang, Z.; Pan, C.; Guo, W.; Chen, C.-Y.; Zhou, Y.; Yu, R.; Wang, Z. L. Tiboelectric-Generator-Driven Pulse Electrodeposition for Micropatterning. Nano Lett. 2012, 12, 4960-4965.

(15) Do, J.-S.; Chen, M.-L. Decolorization of Dye-containing Solutions by Electro-coagulation. J. Appl. Electrochem. 1994, 24, 785-790.

(16) Zhan, X. M; Wang, J. L.; Wen, X. H.; Qian, Y. Indirect Electrochemical Treatment of Saline Dyestuff Wastewater. Environ. Technol. 2001, 22, 1105-1111.

(17) Ma, H.; Wang, B.; Luo, X. Studies on Degradation of Methyl Orange Wastewater by Combined Electrochemical Process. J. Hazard. Mater. 2007, 149, 492-498.

(18) Panizza, M.; Cerisola, G. Electrochemical Oxidation As a Final Treatment of Synthetic Tannery Wastewater. Environ. Sci. Technol. 2004, 38, 5470-5475.

(19) Mohan, N.; Balasubramanian, N.; Basha, C. A. Electrochemical Oxidation of Textile Wastewater and its Reuse. J. Hazard. Mater. 2007, 147, 644-651.

(20) Vecitis, C. D.; Gao, G.; Liu, H. Electrochemical Carbon Nanotube Filter for Adsorption, Desorption, and Oxidation of Aqueous Dyes and Anions. J. Phys. Chem. C. 2011, 115, 3621-3629.

(21) Panizza, M.; Cerisola, G. Electrocatalytic Materials for the Electrochemical Oxidation of Synthetic Dyes. Appl. Catal. B: Environ. 2007, 75, 95-101.

(22) Cross, J. A. Electrostatics: Principles, Problems and Applications; Adam Hilger: Bristol, U.K., 1987.

(23) Nemeth, E.; Albrecht, V.; Schubert, G.; Simon, F. Polymer Triboelectric Charging: Dependence on Thermodynamic Surface Properties and Relative Humidity. J. Electrostat. 2003, 58, 3-16.

(24) Yang, Y.; Long, L.; Zhang, Y.; Jing, Q.; Hou, T.-C.; Wang, Z. L. Self-Powered Magnetic Sensor Based on a Triboelectric Nanogenerator. ACS Nano 2012, 6, 10378-10383.

(25) Wen, S.; Chung, D. D. L. Pyroelectric Behavior of CementBased Materials. Cem. Concr. Res. 2003, 33, 1675-1679.

(26) Chiang, L.; Chang, J.; Wen, T. Indirect Oxidation Effect in Electrochemical Oxidation Treatment of Landfill Leachate. Water Res. 1995, 29, 671-378.

(27) Niu, P.; Hao, J. Fabrication of Titanium Dioxide and Tungstophosphate Nanocomposite Films and Their Photocatalytic Degradation for Methyl Orange. Langmuir 2011, 27, 13590-13597.

(28) Xu, S.; Zhu, Y.; Jiang, L.; Dan, Y. Visible Light Induced Photocatalytic Degradation of Methyl Orange by Polythiophene/ $\mathrm{TiO}_{2}$ Composite Particles. Water Air Soil Pollut. 2010, 213, 151-159. 